\title{
Photostimulable Near-Infrared Persistent Luminescent Nanoprobes for Ultrasensitive and Longitudinal Deep-Tissue Bio-Imaging
}

\author{
Yen-Jun Chuang1 ${ }^{*}$, Zipeng Zhen ${ }^{2,3^{*}}$, Fan Zhang4, Feng Liu'1,5, Jyoti P. Mishra6,7, Wei Tang2,3, Hongmin \\ Chen ${ }^{2,3}$, Xinglu Huang4, Lianchun Wang 6,7 , Xiaoyuan Chen ${ }^{4}$, Jin Xie ${ }^{2,3}$, Zhengwei Pan ${ }^{1,5 凶}$ \\ 1. College of Engineering, University of Georgia, Athens, GA 30602, USA; \\ 2. Department of Chemistry, University of Georgia, Athens, GA 30602, USA; \\ 3. Bio-Imaging Research Center, University of Georgia, Athens, GA 30602, USA; \\ 4. National Institute of Biomedical Imaging and Bioengineering, National Institutes of Health (NIH), Bethesda, MD 20852, USA; \\ 5. Department of Physics and Astronomy, University of Georgia, Athens, GA 30602, USA; \\ 6. Department of Biochemistry and Molecular Biology, University of Georgia, Athens, GA 30602, USA; \\ 7. Complex Carbohydrate Research Center, University of Georgia, Athens, GA 30602, USA. \\ * These authors contributed equally.
}

$\triangle$ Corresponding author: Zhengwei Pan, E-mail: panz@uga.edu. Jin Xie, E-mail: jinxie@uga.edu.

() Ivyspring International Publisher. This is an open-access article distributed under the terms of the Creative Commons License (http://creativecommons.org/ licenses/by-nc-nd/3.0/). Reproduction is permitted for personal, noncommercial use, provided that the article is in whole, unmodified, and properly cited.

Received: 2014.05.2I; Accepted: 2014.05.29; Published: 2014.08.24

\begin{abstract}
In vivo fluorescence imaging suffers from suboptimal signal-to-noise ratio and shallow detection depth, which is caused by the strong tissue autofluorescence under constant external excitation and the scattering and absorption of short-wavelength light in tissues. Here we address these limitations by using a novel type of optical nanoprobes, photostimulable $\mathrm{LiGa}_{5} \mathrm{O}_{8}: \mathrm{Cr}^{3+}$ near-infrared (NIR) persistent luminescence nanoparticles, which, with very-long-lasting NIR persistent luminescence and unique photo-stimulated persistent luminescence (PSPL) capability, allow optical imaging to be performed in an excitation-free and hence, autofluorescence-free manner. $\mathrm{LiGa}_{5} \mathrm{O}_{8}: \mathrm{Cr}^{3+}$ nanoparticles pre-charged by ultraviolet light can be repeatedly ( $>20$ times) stimulated in vivo, even in deep tissues, by short-illumination ( $\sim 15$ seconds) with a white light-emitting-diode flashlight, giving rise to multiple NIR PSPL that expands the tracking window from several hours to more than 10 days. Our studies reveal promising potential of these nanoprobes in cell tracking and tumor targeting, exhibiting exceptional sensitivity and penetration that far exceed those afforded by conventional fluorescence imaging.
\end{abstract}

Key words: $\mathrm{LiGa}_{5} \mathrm{O}_{8}: \mathrm{Cr}^{3+}$ nanoparticles, near-infrared, persistent luminescence, photostimulated persistent luminescence, in vivo imaging.

\section{Introduction}

Macroscopic optical imaging is becoming an essential tool in biomedical research and drug development, owing to its advantages of high-throughput, non-invasive, non-radioactive, easy-to-operate, and cost-effective detection [1-4]. Most optical imaging techniques work by tracing fluorescence signals from optical probes. To obtain high-quality imaging, tre- mendous efforts have been spent on finding novel optical probes, made of either organic molecules [5, 6], proteins [7-9], or nanoparticles [10-14]. Much attention has been paid to materials with high quantum yields, good biocompatibility, robust chemical- and photo-stability, low toxicity, and most importantly, near-infrared (NIR) emission [12, 13], because NIR 
light (particularly in the $650-950 \mathrm{~nm}$ spectral range) has a relatively low level of tissue interference $[5,15]$. Despite some progress, fluorescence imaging suffers from a suboptimal signal-to-noise $(\mathrm{S} / \mathrm{N})$ ratio and a shallow detection depth $(<1 \mathrm{~cm})$ [1]. These drawbacks are mainly associated with tissue autofluorescence, which is caused by the fluorescence/scattering from tissues under constant external irradiation [16, 17]. Thus, ideally, an optical probe that can illuminate without external excitation would be free of autofluorescence and hence, enable significantly improved imaging sensitivity and depth. The related research in this area, however, has been very limited. Recently Rao et al. coupled a miniature light source - a renilla luciferase protein - onto a quantum dot $[3,18]$ or a semiconductor polymer nanoparticle [19], so that the nanoparticle fluorophore could be illuminated by bioluminescence resonance energy transfer, or BRET. Though conceptually interesting, the applications of these BRET-based technologies in translational research are limited, with possible concerns over the challenges of making the probes, in vivo stability, heavy-metal induced toxicity, and dependence on the distribution of the luciferase substrate [16].

To address the limitations of fluorescence-based imaging, luminescent nanoparticles capable of emitting red/NIR persistent luminescence were recently used as the optical probes in bioimaging $[17,20]$. Persistent luminescence, also called afterglow, is a phenomenon whereby luminescence can last for a long time, typically for time scales on the order of hours to days for bulk materials, after the stoppage of the excitation [21, 22]. Due to the small size of the imaging probes (typically $<100 \mathrm{~nm}$ ) and the small dosage (in micro-gram level) permitted, however, the longevity of the persistent luminescence in vivo is short. The longest observation time for subcutaneously injected persistent luminescent nanoparticles was $\sim 15 \mathrm{~h}$ [20], while the in vivo distribution of intravenously injected nanoparticles was followed in real-time for only 0.5 to $7.5 \mathrm{~h}[17,20]$. Such time spans are barely sufficient for imaging molecular targets, not to mention cell tracking, where a detection window of days or even weeks is required [23]. Since persistent luminescent materials are generally effective to ultraviolet (UV) excitation which has short tissue penetration and is harmful to normal tissues, it is not possible to recharge the nanoprobes using UV light once the probes are inside a living subject. Thus, limited luminescence longevity is the major obstacle to the use of persistent luminescence in bioimaging. Moreover, attempts of using persistent luminescent nanoparticles for tumor targeting only received very limited success; while luminescence signal was observed in ex vivo imaging of collected tumors, almost no luminescence signals could be detected in tumor areas in vivo after $20 \mathrm{~min}$ post-injection $[17,20]$.

We recently developed a new NIR persistent luminescent material - trivalent chromium $\left(\mathrm{Cr}^{3+}\right)$ doped lithium gallate with a chemical formula of $\mathrm{Li}$ $\mathrm{Ga}_{5} \mathrm{O}_{8}: \mathrm{Cr}^{3+}$ (hereafter referred to as LGO:Cr) [24]. Bulk LGO:Cr exhibits a super-long NIR persistent luminescence (peaking at $716 \mathrm{~nm}$ ) of more than 1,000 h after minutes of UV irradiation, which is the longest persistent luminescence reported to date. Remarkably, the LGO:Cr phosphor is also a superb photostimulable storage medium - the energy stored in the deep traps of the material can be liberated by photo-stimulation as intense NIR persistent luminescence signal, for multiple times ( $>20$ times) over a very-long period of time $(>2,000 \mathrm{~h})$, by short exposure (tens of seconds) to a white light-emitting diode (LED) illumination - a new phenomenon that we referred to as photo-stimulated persistent luminescence (PSPL) [24]. By taking advantage of this new NIR PSPL phenomenon, in this article we report that the LGO:Cr phosphor in the form of nanoparticles can act as an ideal self-illuminating NIR optical nanoprobe that allows for in vivo tracking for more than 10 days when injected subcutaneously and for more than 3 days when injected intravenously. These time spans are about 1 to 3 orders of magnitude longer than that achieved in the previous persistent luminescence-based imaging. This long-term tracking capability, along with the exceptional sensitivity and deep penetration depth, breaks several of the most constraining factors of optical imaging, and is expected to open many new avenues for both biomedical research and in vivo clinical applications.

\section{Materials and Methods}

Synthesis of LGO:Cr nanoparticles. The LGO:Cr nanoparticles were synthesized by a sol-gel method using lithium nitrate $\left(\mathrm{LiNO}_{3}\right)$, gallium nitrate $\left(\mathrm{Ga}\left(\mathrm{NO}_{3}\right)_{3}\right)$ and chromium nitrate $\left(\mathrm{Cr}\left(\mathrm{NO}_{3}\right)_{3}\right)$ as precursors, followed by calcination and then wet mechanical grinding. A solution was prepared by dissolving $0.19 \mathrm{~g} \mathrm{LiNO}_{3}$ (99\%), $5.0 \mathrm{~g} \mathrm{Ga}\left(\mathrm{NO}_{3}\right)_{3}(99.999 \%)$ and $1.65 \mathrm{mg} \mathrm{Cr}\left(\mathrm{NO}_{3}\right)_{3}(98.5 \%)$ in $44 \mathrm{~mL}$ methanol. The solution was stirred for several hours on a magnetic stirrer. During the stirring process, a chelating agent, $5.0 \mathrm{~mL}$ acetylacetone $(>99 \%)$, was added into the solution to form chelate complexes. Aqueous ammonium solution ( $28 \mathrm{vol}$ \%) was also added to stabilize the complexes and to adjust the $\mathrm{pH}$ of the sol to 8.4. When a homogeneous sol was formed, the solution was heated to $90^{\circ} \mathrm{C}$ for $5 \mathrm{~h}$ to form dry gel. The dry gel was then calcinated in a muffle furnace at $1100{ }^{\circ} \mathrm{C}$ for $3 \mathrm{~h}$ to form LGO:Cr nanoparticles with desired optical properties. Since most of the as-calcinated 
particles are larger than $200 \mathrm{~nm}$ due to sintering, to obtain small nanoparticles in large scale (gram scale) the as-calcinated particles were wet grinded using a ball milling equipment (JBM-B035, Just Nanotech Co.) in 2-propanol medium. After filtration and centrifugation, LGO:Cr nanoparticles with diameters in the range of 50-100 nm were obtained.

Nanoparticle characterizations. The crystal structure of the LGO:Cr nanoparticles was analyzed using a PANalytical X'Pert PRO powder $x$-ray diffractometer with $\mathrm{Cu} \mathrm{Ka} 1$ radiation $(\lambda=1.5406 \AA)$. The morphology and size of the nanoparticles were studied using a JEOL JEM 1210 transmission electron microscope (TEM). The optical properties were measured using a Horiba FluoroLog-3 spectrofluorometer equipped with a $450 \mathrm{~W}$ xenon arc lamp and a R928P photomultiplier tube $(250-850 \mathrm{~nm})$. All spectra were corrected for the optical system responses. Appropriate optical filters were used to avoid stray light. An ATN PVS-14 Generation III night vision monocular was used to monitor the "brightness" of NIR persistent luminescence and NIR PSPL signals, as well as to take NIR images. The monitoring and imaging experiments were conducted in darkroom. Before all the spectral measurements and imaging, the samples were heat-treated in a muffle oven at $400{ }^{\circ} \mathrm{C}$ for 20 min to completely empty the electron traps.

Coating LGO:Cr nanoparticles with polyethylenimine (PEI). LGO:Cr nanoparticles were dispersed in a solution of $12.5 \mathrm{wt} \%$ PEI (MW: 25000, Aldrich) and then stirred for two days. The raw PEI-LGO:Cr nanoparticles were washed with deionized water for 3 times and collected by centrifugation. The purified PEI-LGO:Cr nanoparticles were dispersed in $1 \times$ phosphate buffered saline (PBS, $\mathrm{pH} 7.4$ ), and sterilized by UV irradiation in a laminar flow hood for 72 h.

Viability assays. The cytotoxicity of the PEI-LGO:Cr nanoparticles were evaluated on three cell lines: 4T1 murine breast cancer cells, RAW264.7 murine macrophage cells, and murine embryonic stem cells (ESCs). The impacts of PEI-LGO:Cr nanoparticles on the renewal and differentiation of mouse ESCs were studied by analyzing three key biomarkers: Nanog, Nestin and Sox17. The detailed procedure for viability measurements is described in the Supplementary Material.

Cell labeling. Sterilized PEI-LGO:Cr nanoparticles were added into cell growth medium and incubated with cells at $37^{\circ} \mathrm{C}$ for $24 \mathrm{~h}$. Typically, a particle concentration of $100 \mu \mathrm{g} / \mathrm{mL}$ was used. For in vitro imaging, LGO:Cr-labeled cells were fixed with 70\% ethanol at $4{ }^{\circ} \mathrm{C}$ for $12 \mathrm{~h}$. For in vivo imaging, UV pre-irradiated PEI-LGO:Cr nanoparticles were used, and the cell culture and inoculation were performed in the dark or in a safelight condition to avoid energy loss.

Cell differentiation and thin-section TEM imaging. $4 \mathrm{~T} 1$ cells $\left(1.0 \times 10^{6}\right.$ cells $\left./ \mathrm{mL}\right)$ were exposed to PEI-LGO:Cr nanoparticles $(100 \mu \mathrm{g} / \mathrm{mL})$ and co-incubated in cell culture flask for 2 days using RPMI-1640 medium containing 10\% fetal bovine serum. The cells were then collected by treatment with trypsin. For successive culturing, the cells from the $1^{\text {st }}$ generation were further cultured in medium without the presence of additional PEI-LGO:Cr nanoparticles, till the $3^{\text {rd }}$ generation. These three generations of cells were then subjected to thin-section TEM imaging. The detailed procedure used to prepare thin-section samples is described in the Supplementary Material.

Preparation of $c(R G D y K)$-conjugated LGO:Cr nanoparticles. LGO:Cr nanoparticles were dispersed in ethanol to a concentration of $1 \mathrm{mg} / \mathrm{mL}$. Polyvinylpyrollidone (PVP, MW $=40 \mathrm{k}$, Sigma) was added into the solution, and the mixture was magnetically stirred in the dark for $48 \mathrm{~h}$. The resulting PVP-LGO:Cr nanoparticles were collected via centrifugation and washed with ethanol for 3 times. The purified PVP-LGO:Cr nanoparticles were added to a human serum albumin (HSA) solution ( $3 \mathrm{mg} \mathrm{HSA} / \mathrm{mL}$ ), and the mixture was incubated for $4 \mathrm{~h}$. HSA was adsorbed onto the surface of LGO:Cr nanoparticles, and the product was collected by centrifugation and washed with water for 3 times. For bioconjugation, the HSA-coated LGO:Cr nanoparticles were first reacted with bis(sulfosuccinimidyl) suberate (BS33, Thermo Scientific) in a $50 \mathrm{mM}$ borate buffer solution ( $\mathrm{pH} 8.0$ ) for $30 \mathrm{~min}$ at room temperature. The particles were purified by centrifugation and re-dispersed in PBS (pH 7.4). c(RGDyK) was added to the solution, and the reaction was conducted at room temperature for 2 $h$. The products were purified by centrifugation and re-dispersed in PBS.

Bioluminescence microscopic study. An Olympus LV200 bioluminescence microscope was used to take luminescence images of PEI-LGO:Cr labeled 4T1 cells and PEI-LGO:Cr labeled firefly luciferase (f-luc) 4T1 cells in both bright-field and bioluminescence modes. For PEI-LGO:Cr-labeled f-luc 4T1 cells, imaging was first performed by setting the emission filter to "open" (without the use of any emission filter). Subsequently, the cells were UV-irradiated for $5 \mathrm{~min}$, and imaging was conducted again using a "Cy5.5" emission filter (HQ725/50M). All the images were processed using CellSens Dimension (Version 1.71, Olympus Corporation, Tokyo).

Imaging on an IVIS Lumina II imaging system. The images were acquired in the bioluminescence mode for LGO:Cr nanoparticles and f-luc, and in the fluorescence mode for quantum dots (QDs). For NIR 
persistent luminescence-based imaging, LGO:Cr nanoparticles or cells/mouse organs bearing LGO:Cr nanoparticles were irradiated by a $254 \mathrm{~nm}$ UV lamp for $15 \mathrm{~min}$. For NIR PSPL-based imaging, mice bearing LGO:Cr nanoparticles were illuminated by an Olight SR51 white LED flashlight (900 lumens) for 15 $\mathrm{s}$, with the flashlight placed $6 \mathrm{~cm}$ above the mice. For QDs-based imaging, mice bearing QDs were irradiated by $570 \mathrm{~nm}$ light while imaging. All the images were processed using a Living Image software (Version 4.3.1 SP1, PerkinElmer) with "binning" set at 4 and "smooth" set at $5 \times 5$.

Inoculation of PEI-LGO:Cr nanoparticles into mouse brain. Nude mice were anesthetized with 1-2\% isoflurane mixed with pure oxygen via a nose cone. The head was positioned and immobilized in a stereotaxic frame, and a small opening was made in the skull and dura overlying the somatosensory cortex. About $25 \mu \mathrm{g}$ UV pre-irradiated PEI-LGO:Cr nanoparticles were intracranially injected into the right frontal lobe at coordinates $1.5 \mathrm{~mm}$ lateral from the bregma, $0.5 \mathrm{~mm}$ anterior, and $1 \mathrm{~mm}$ intraparenchymal through a $10 \mu \mathrm{L}$ Hamilton syringe.

Cell tracking and active targeting. The $4 \mathrm{~T} 1$ tumor model was established by subcutaneously inoculating $5 \times 10^{5} 4 \mathrm{~T} 1$ cells to the shoulder of a nude mouse. For the cell tracking study, PEI-LGO:Cr nanoparticles were UV pre-irradiated by a $254 \mathrm{~nm}$ UV lamp for $15 \mathrm{~min}$, and then loaded into RAW264.7 cells for a $48 \mathrm{~h}$ incubation. $\sim 1.0 \times 10^{6}$ LGO:Cr-bearing RAW264.7 cells were intravenously injected into a 4T1 tumor bearing mouse. For the active tumor targeting study, $0.5 \mathrm{mg}$ c(RGDyK)-conjugated LGO:Cr nanoparticles were irradiated by a $254 \mathrm{~nm}$ UV lamp for 15 min, and then intravenously injected into a $4 \mathrm{~T} 1$ tumor bearing mouse.

Biodistribution and histology studies. After the cell tracking and active targeting experiments, mice were sacrificed. Tumor and major organs including the liver, lung, spleen, kidney, heart, and brain were harvested, UV-irradiated, and then subjected to imaging on an IVIS imaging system in the bioluminescence mode. The tumors were then snap-frozen, cut into $6-8 \mu \mathrm{m}$ thick sections, mounted on a coverslip, and fixed in acetone at room temperature for $2 \mathrm{~h}$. The sections were gently washed with PBS ( $\mathrm{pH}$ 7.4) for 3 times, irradiated by a $254 \mathrm{~nm}$ UV lamp for $5 \mathrm{~min}$, and subjected to imaging on an Olympus LV200 microscope.

All the animal studies were performed according to a protocol approved by the Institutional Animal
Care and Use Committee (IACUC) of the University of Georgia.

\section{Results and Discussion}

Properties of LGO:Cr nanoparticles. The as-prepared LGO:Cr nanoparticles are single-crystalline with a cubic crystal structure (Figure S1 in the Supplementary Material), and have diameters ranging from 50 to $100 \mathrm{~nm}$ (Figure 1A). Like their bulk counterparts [24], the LGO:Cr nanoparticles exhibit strong NIR photoluminescence, NIR persistent luminescence, and NIR PSPL, as shown in Figures 1B-D, respectively. Specifically, the particles can be effectively excited by light of a broad range of wavelengths (from $\sim 250 \mathrm{~nm}$ to $\sim 660 \mathrm{~nm}$ ) to emit an intense and narrow photoluminescence band peaking at $716 \mathrm{~nm}$ (Figure 1B), which is attributed to the spin-forbidden ${ }^{2} E \rightarrow{ }^{4} A_{2}$ transition of $\mathrm{Cr}^{3+}$ ions [24, 25]. After charged with a $254 \mathrm{~nm}$ UV lamp for $15 \mathrm{~min}$, the energy (in the form of electrons) stored in the traps (including shallow traps and deep traps [24]) of the nanomaterials can sustain NIR persistent luminescence for up to 100 h (Figure 1C and Figures S2A-C). When the persistent luminescence signal becomes very weak, owing to the emptying of the shallow traps, short illumination (e.g., 15 s) with a white LED flashlight can stimulate the release of some of the electrons in the deep traps to refill the emptied shallow traps, resulting in the rejuvenation of the persistent luminescence (Figure 1D), via a PSPL mechanism [24]. The resulting PSPL signal can last for up to $1 \mathrm{~h}$, and the process can be repeated multiple times ( $>10$ times) over a period of several hundred hours (Figures S2D-F). The profiles of the persistent luminescence emission spectrum (inset of Figure 1C) and the PSPL emission spectrum (inset of Figure 1D) are almost identical to that of the photoluminescence spectrum (Figure 1B), indicating that both NIR persistent luminescence and NIR PSPL originate from the $\mathrm{Cr}^{3+}$ emitting centers. It should be noted that for the PSPL process to occur, the nanoparticles must be pre-irradiated by UV light; without the UV pre-irradiation, the white LED illumination does not produce NIR persistent luminescence signals (the grey curve in Figure 1D), because the energy of the white light is too low to photoionize the localized electrons from $\mathrm{Cr}^{3+}$ to the conduction band to fill the traps $[22,24]$. It is also worth noting that LGO:Cr nanoparticles in aqueous solutions exhibit the same persistent luminescence and PSPL properties as they do in dry powder form, as shown in Supplementary Material: Figure S3. 

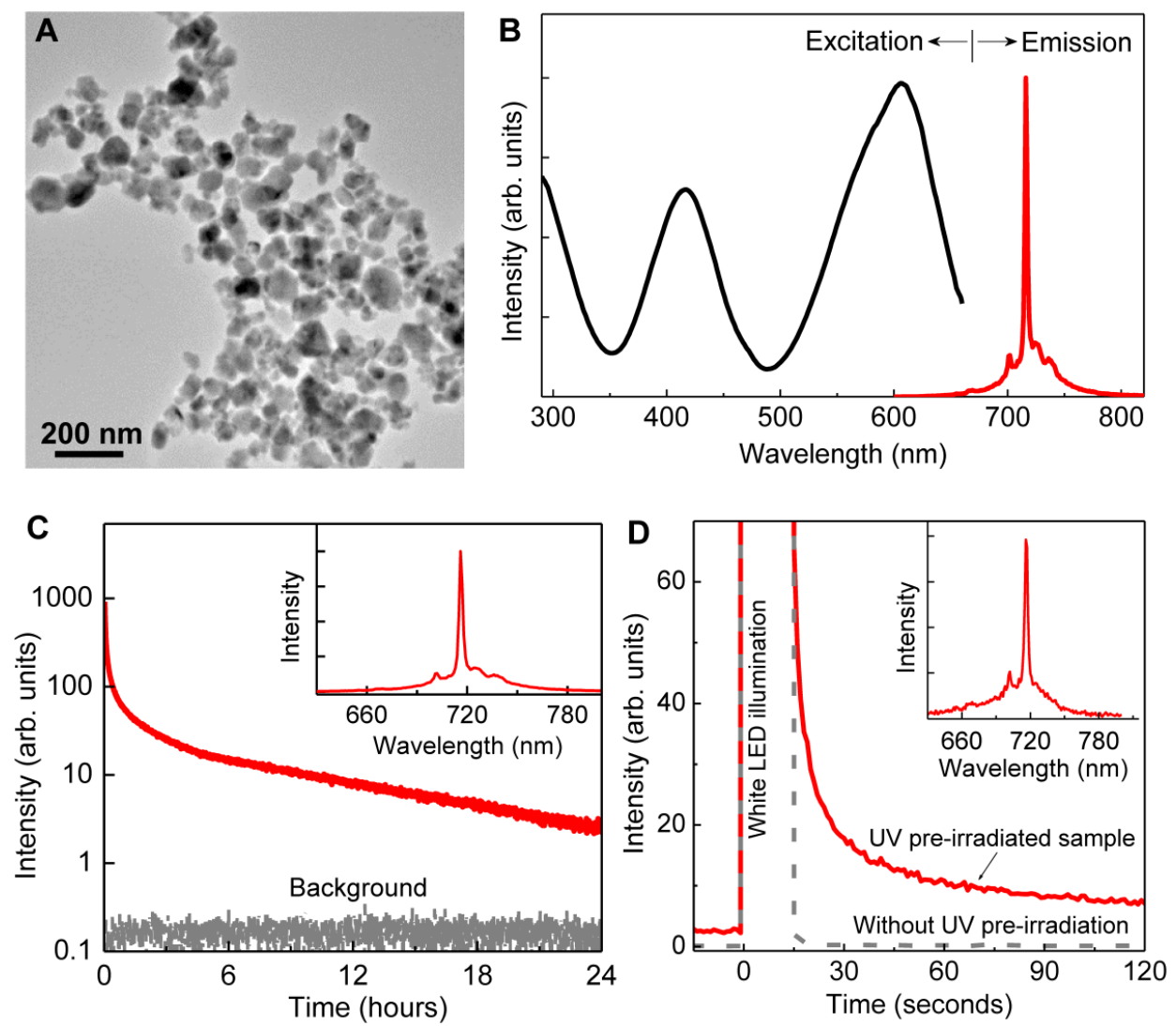

Figure I. Morphology and luminescence properties of LGO:Cr nanoparticles. (A) TEM image of LGO:Cr nanoparticles. (B) Normalized excitation and emission spectra of LGO:Cr nanoparticles at room temperature. The excitation spectrum was obtained by monitoring at $716 \mathrm{~nm}$ emission. (C) NIR persistent luminescence decay curve of LGO:Cr nanoparticles monitored at $716 \mathrm{~nm}$ after irradiation by a $254 \mathrm{~nm}$ UV lamp for $15 \mathrm{~min}$. The bottom curve is the background. The inset is the persistent luminescence emission spectrum recorded at $10 \mathrm{~s}$ after the UV irradiation. (D) PSPL decay curves monitored at $716 \mathrm{~nm}$ emission after stimulation by a white LED flashlight for $15 \mathrm{~s}$. The red solid curve was acquired on a $24 \mathrm{~h}$ decayed sample (pre-irradiated by a $254 \mathrm{~nm}$ UV lamp for $15 \mathrm{~min}$ ), while the grey dash line curve was recorded on a bleached sample (without UV pre-irradiation). The inset is the PSPL emission spectrum of the $24 \mathrm{~h}$ decayed sample, which was recorded at $10 \mathrm{~s}$ after the stimulation.

Viability assays. For biomedical imaging applications, we coated the LGO:Cr nanoparticles with a layer of polyethylenimine (PEI, MW: 25000), a polycation polymer that is widely used in cell labeling and gene transfection because it facilitates cell internalization [26, 27]. The cytotoxicity of the resulting PEI-coated LGO:Cr (PEI-LGO:Cr) nanoparticles was studied with a panel of cell lines, including 4T1 (murine breast cancer), RAW264.7 (murine macrophage), and murine embryonic stem cells (ESCs). For all the cell lines, we observed $>80 \%$ viability in the tested concentration range (up to $100 \mu \mathrm{g} / \mathrm{mL}$; Supplementary Material: Figure S4), indicating a good tolerance of the cells to the particles. For ESCs, we also studied the impact of the labeling on cell renewal and differentiation by analyzing three key biomarkers, i.e., Nanog, Nestin and Sox17, and found the influence insignificant (Supplementary Material: Figure S5). Such good biocompatibility is not unexpected given that all the three constituting metal elements - lithium, gallium and trivalent chromium - are known to have relatively low toxicity profiles. Lithium is regarded as an essential nutrient for the human body [28], a trace amount of trivalent chromium in diet has no adverse effect to human health [29], and gallium-based probes, for instance gallium-67 citrate, were once widely used for cancer diagnosis and staging in the clinic [30].

Cell labeling and individual cell imaging. For all the studied cell lines, incubation with PEI-LGO:Cr nanoparticles overnight results in good internalization and thus good labeling efficiency. Figure 2A is a thin-section TEM image of a single 4T1 cell labeled with PEI-LGO:Cr nanoparticles. Many nanoparticles were accumulated in the cell endosomes/lysosomes, suggesting that the particle uptake was mainly mediated by endocytosis. These nanoparticles remained stable in endosomes/lysosomes and were able to be carried over to the daughter cells (Supplementary Material: Figure S6). The acidic lysosomal environment has no detectable impact on the particles' morphology and, as the long-duration in vivo tracking experiments demonstrated below, the particles' NIR performance.

The labeling efficiency of PEI-LGO:Cr nanoparticles to 4T1 cells (fixed) was also studied using an Olympus LV200 bioluminescence microscope, which takes images based on glow-in-the-dark signals. After 
pre-charged with a $254 \mathrm{~nm}$ UV lamp for $5 \mathrm{~min}$, almost all the cells were clearly visualized (Figure 2B-D) owing to good cell labeling efficiency and complete elimination of the background noise in the imaging system. The cells remained detectable for more than 30 min (Supplementary Material: Figure S7). To our best knowledge, this is the first case of cell imaging based on persistent luminescence signals.

Owing to the narrow emission band in the NIR, the LGO:Cr nanoparticles can work along with visible bioluminescence probes, like firefly luciferase (f-luc), for multiplexed imaging. To test this, we labeled 4T1 cells that stably express f-luc (f-luc-4T1) with LGO:Cr nanoparticles, and imaged the dually-labeled cells on a LV200 microscope. To differentiate the LGO:Cr nanoparticles' NIR persistent luminescence ( 650-850 $\mathrm{nm}$ peaking at $716 \mathrm{~nm}$ ) from the f-luc's visible bioluminescence $(\sim 460-670 \mathrm{~nm}$ peaking at $557 \mathrm{~nm}$ at room temperature) [31], a Cy5.5 filter was used when collecting the NIR signals (only 698-752 nm light can pass the filter). Indeed, luminescence from both sources was clearly visualized, as the images shown in Figures 2E and 2F. While overlaps were observed across the scope, discrepancies were also found in many cells, suggesting good discrimination of the two signals (Figures $2 \mathrm{G}$ and $2 \mathrm{H}$ ). Notably, the intensity of the NIR persistent luminescence from LGO:Cr nanoparticles is comparable to that of the bioluminescence from f-luc; the latter is the most sensitive optical method for cell tracking utilized to date [31,32].

Detection sensitivity and detection depth. The good sensitivity of the LGO:Cr nanoparticles-based
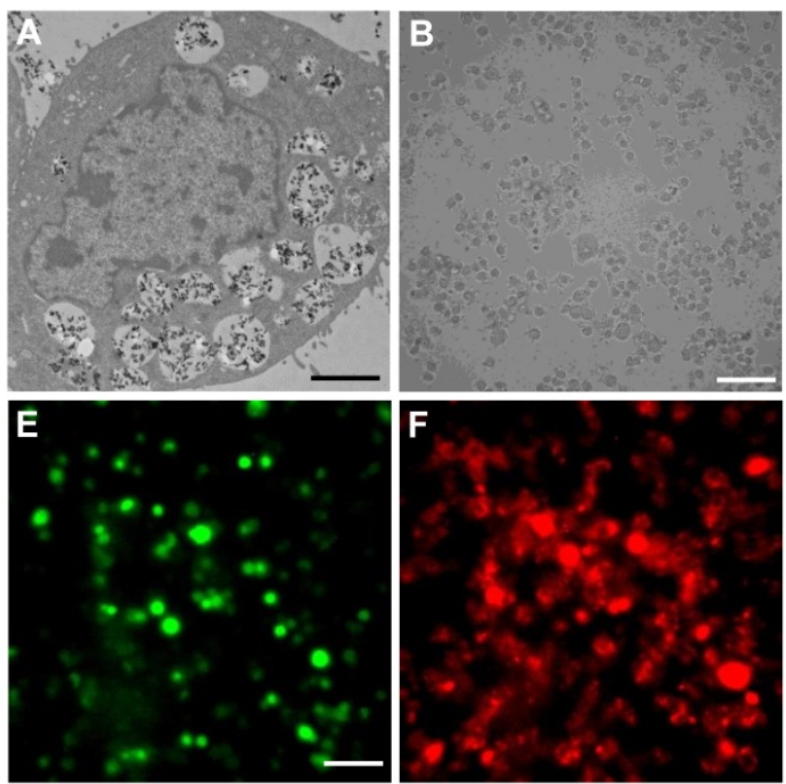

imaging was further revealed through in vivo studies. We subcutaneously implanted elevated numbers $(\sim 5$, 50, 100, 500 and 1,000) of PEI-LGO:Cr nanoparticles-labeled RAW264.7 cells onto the back of a nude mouse and subjected the animal to imaging on an IVIS Lumina II imaging system in the "bioluminescence" mode, as shown in Figure 3A. As few as $\sim 5$ cells can be clearly visualized. This sensitivity is comparable to f-luc-based cell tracking [32], but several orders of magnitude higher than that of QDs [33] and organic dyes [34]. The advantage of such a high sensitivity was clearly manifested in a comparison study, where the same amount ( $32 \mu \mathrm{g})$ of LGO:Cr nanoparticles and QDs (emission at $705 \mathrm{~nm}$ ) were respectively inoculated onto the backs of two nude mice (Figures 3B and 3D). Despite a much lower number of photons generated, in dorsal view the S/N ratio with LGO:Cr (in the "bioluminescence" mode) was significantly higher than that with QDs (in the "fluorescence" mode) (Figure 3F). This is attributed to the elimination of external excitation and along with it, the autofluorescence, in LGO:Cr nanoparticles-based imaging. The benefit of the elimination of autofluorescence became overwhelmingly significant when the mice were viewed in the ventral position. While the persistent luminescence signal from LGO:Cr nanoparticles was able to be clearly visualized through the animal body (Figure 3C), the fluorescence signal from QDs was submerged by the overwhelming autofluorescence signals and could barely be distinguished by the IVIS imaging system (Figure 3E).

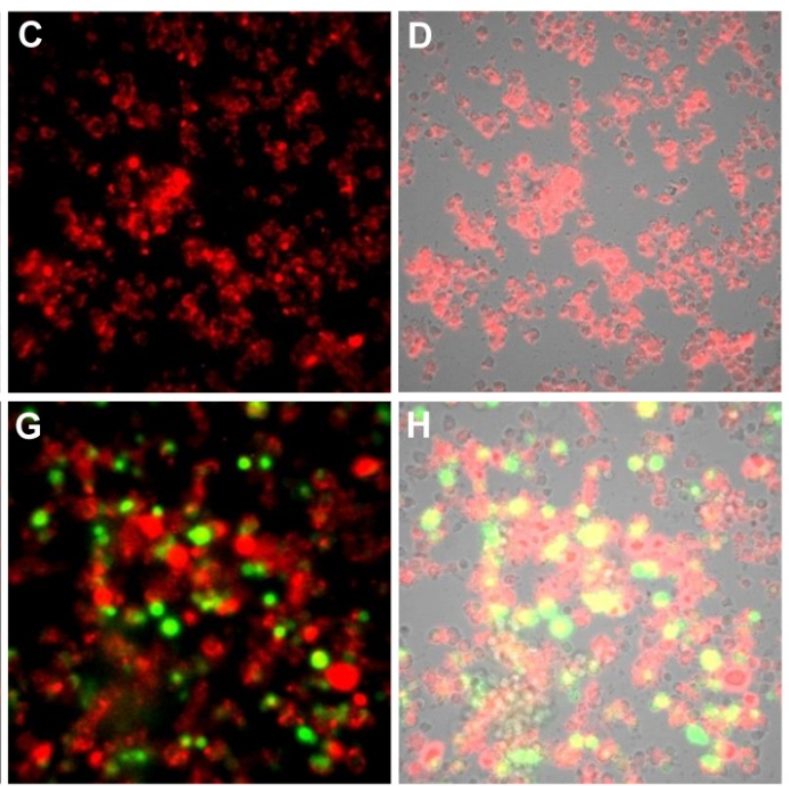

Figure 2. TEM and luminescence microscopic studies of PEI-LGO:Cr nanoparticles-labeled 4TI cells. (A) Thin-section TEM image of a 4TI cell labeled with PEI-LGO:Cr nanoparticles. The nanoparticles were accumulated in cell endosomes/lysosomes. Scale bar, $2 \mu \mathrm{m}$. (B) Bright-field image of PEI-LGO:Cr labeled 4TI cells (fixed). Scale bar, I00 $\mu \mathrm{m}$. (C) NIR persistent luminescence image of the cells in (B) after irradiation by a $254 \mathrm{~nm}$ UV lamp for $5 \mathrm{~min}$. The image was acquired $20 \mathrm{~s}$ after the irradiation. (D) Merged image of (B) and (C). (E) Bioluminescence image of PEI-LGO:Cr-labeled f-luc-4TI cells. The cells were incubated with D-luciferin ( $150 \mu \mathrm{g} / \mathrm{mL}) 10 \mathrm{~min}$ before the imaging. The image was taken by setting the emission filter as "open". The signals were from f-luc. Scale bar, $100 \mu \mathrm{m}$. (F) NIR persistent luminescence image of the cells in (E) after irradiation by a $254 \mathrm{~nm}$ UV lamp for $5 \mathrm{~min}$. A Cy5.5 emission filter was used. (G) Merged image of $(E)$ and $(F)$. (H) Merged image of $(G)$ and the corresponding bright-field image (not shown individually here). The images in (B), (C), (E), and (F) were taken on an Olympus LV200 bioluminescence microscope. The exposure time in $(C),(E)$ and $(F)$ is I min. 

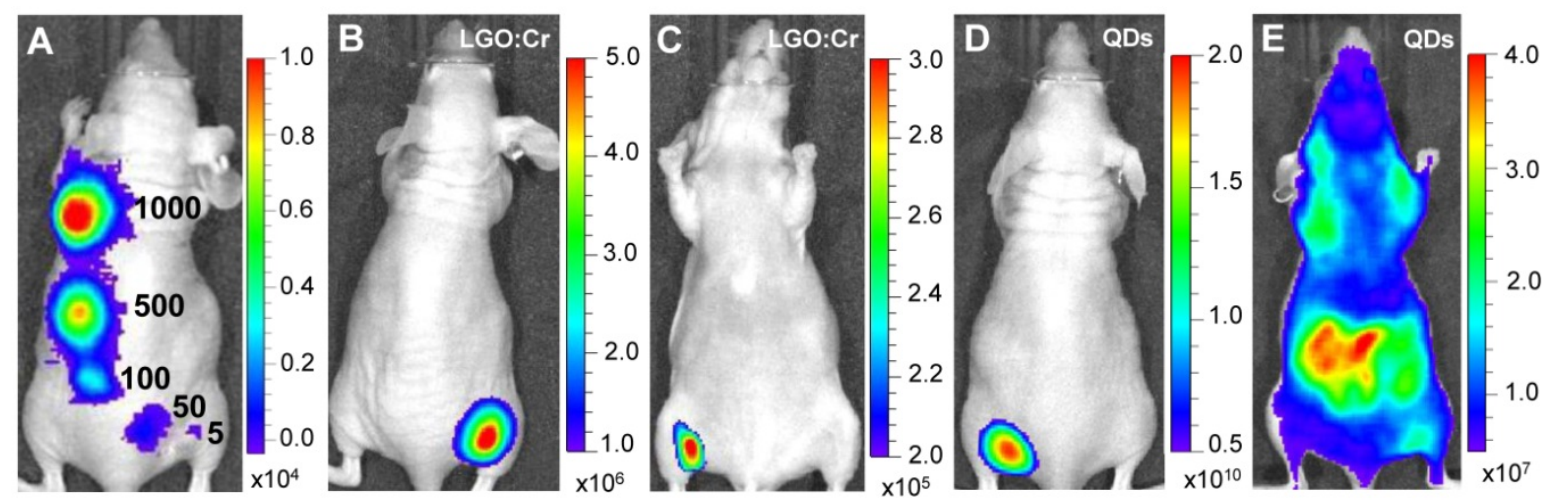

$\mathbf{F}$

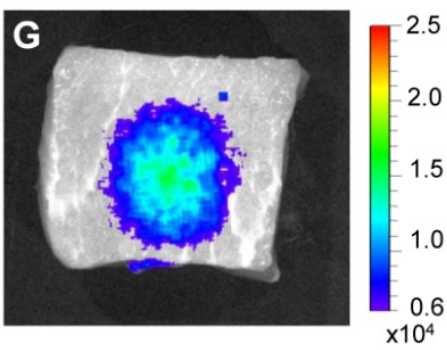

Figure 3. Detection sensitivity and detection depth of LGO:Cr nanoparticles-based imaging. (A) Detection sensitivity of PEI-LGO:Cr-based cell labeling. Elevated numbers ( 5, 50, I00, 500, and I,000) of PEI-LGO:Cr labeled RAW264.7 cells were subcutaneously injected onto the back of a nude mouse. The cells were fixed and irradiated by a 254 nm UV lamp for 5 min before the injection. $(B, C)$ Dorsal and ventral views, respectively, of a nude mouse subcutaneously implanted with $32 \mu \mathrm{g} U V$ pre-irradiated PEI-LGO:Cr nanoparticles. (D,E) Dorsal and ventral views, respectively, of a nude mouse subcutaneously implanted with $32 \mu \mathrm{g}$ QDs (emission at $705 \mathrm{~nm}$ ). The QDs were excited by $570 \mathrm{~nm}$ light while imaging. (F) S/N ratios in (B) and (D). (G) Detection depth study using pork as a model tissue. $15 \mu \mathrm{g}$ UV pre-irradiated LGO:Cr nanoparticles were injected into $2.5 \mathrm{~cm}$ depth inside the pork, and the image was taken at 10 min after the injection. The images were taken on an IVIS imaging system in the bioluminescence mode for LGO:Cr nanoparticles and in the fluorescence mode for QDs. The exposure time is 5 min in (A), 2 min in $(B),(C)$ and $(G)$, and $I s$ in $(D)$ and $(E)$.

The ventral view image in Figure 3C evidently reveals the exceptional tissue penetration depth of the LGO:Cr nanoparticles-based imaging, and such deep penetration has seldom been achieved optically $[1,5$, 15]. In addition to a clean background, the deep penetration is also credited to the luminescence in the NIR spectral region, where the tissues are relatively transparent $[5,15]$. This great tissue penetration is further evidenced in a comparison between LGO:Cr nanoparticles and f-luc under the same excitation-free, bioluminescence imaging mode, as shown in Supplementary Material: Figure S8. Briefly, we subcutaneously implanted the same amount of LGO:Cr nanoparticles and f-luc dually-labeled $4 \mathrm{~T} 1$ cells $\left(\sim 1 \times 10^{6}\right.$ cells) onto the backs of the hind limbs of two nude mice. In dorsal view, the intensities of the f-luc's visible bioluminescence (peaking at $612 \mathrm{~nm}$ at $37^{\circ} \mathrm{C}$ ) and the LGO:Cr nanoparticles' NIR persistent luminescence are comparable. In ventral view, however, the intensity of LGO:Cr persistent luminescence is about an order of magnitude higher than that of f-luc bioluminescence, showing greater transmission of the NIR light than the visible light through tissues. The penetrating power of the NIR light was also investigated in a phantom study using pork as a model tissue. When $15 \mu \mathrm{g}$ UV pre-irradiated LGO:Cr nanoparticles were injected into $2.5 \mathrm{~cm}$ depth inside the pork (thickness of the pork, $\sim 5 \mathrm{~cm}$ ), the NIR persistent luminescence signals from the LGO:Cr nanoparticles can be readily detected by an IVIS imaging system (Figure 3G) and the signals remained detectable for more than $30 \mathrm{~min}$ (Supplementary Material: Figure S9A-D). This penetration depth is greater than that afforded by fluorescence imaging $(<1 \mathrm{~cm})$ and bioluminescence imaging $(<2 \mathrm{~cm})[1]$.

Longitudinal monitoring based on LGO:Cr nanoparticles' PSPL property. As aforementioned, a major restriction of current persistent luminescence-based imaging is its short observation window (up to $15 \mathrm{~h}$ for subcutaneous injection and up to $7.5 \mathrm{~h}$ for intravenous injection) $[17,20]$, which needs to be significantly increased in order to meet the demand of longitudinal imaging such as cell tracking which often needs a window of days or even weeks [23]. This longitudinal tracking demand can be perfectly satisfied by utilizing the LGO:Cr nanoparticles' unique PSPL property. For LGO:Cr nanoparticles-bearing 4T1 cells $\left(\sim 2.5 \times 10^{7}\right.$ cells $)$ subcutaneously implanted into a mouse, the initial NIR persistent luminescence signals remained detectable for more than $4 \mathrm{~h}$ (Figure 4A). When the dimming animal was illuminated with a white LED flashlight (Olight SR51, 900 lumens) for 15 $\mathrm{s}$, the NIR emission from the LGO:Cr nanoparticles in vivo was immediately recovered (Figure $4 \mathrm{~B}$ ), and the resulting PSPL signals remained detectable for $\sim 30$ min. Such a PSPL process can be repeated more than 20 times before depleting the energy stored in the deep traps of the LGO:Cr nanoparticles. This extends 
the tracking window from several hours to more than 10 days. Although the PSPL intensity dropped as the number of stimulations increased (Figure 4B), the PSPL signals remained detectable even on day 10, at which time a still high $\mathrm{S} / \mathrm{N}$ ratio of 24.6 was obtained. Note that an even longer tracking window, e.g., one month, is expected if a less frequent stimulation scheme is adopted [24]. More significantly, owing to the high power of the Olight SR51 flashlight, the white LED illumination can not only stimulate LGO:Cr nanoparticles located superficially but also those positioned deep beneath the skin. This was proven in a separate study where $\sim 25 \mu \mathrm{g}$ UV pre-irradiated PEI-LGO:Cr nanoparticles were implanted into the brain of a mouse. As expected, strong and repeated PSPL signals were observed upon white LED stimulations, as shown in Figure 5 and Supplementary Material: Figure S10. The effectiveness of white LED stimulation was further demonstrated using a pork model; PSPL signals from $15 \mu \mathrm{g}$ LGO:Cr nanoparticles located at $2.5 \mathrm{~cm}$ deep can be readily elicited by short-time white light illumination, as shown in Supplementary Material: Figure S9E.
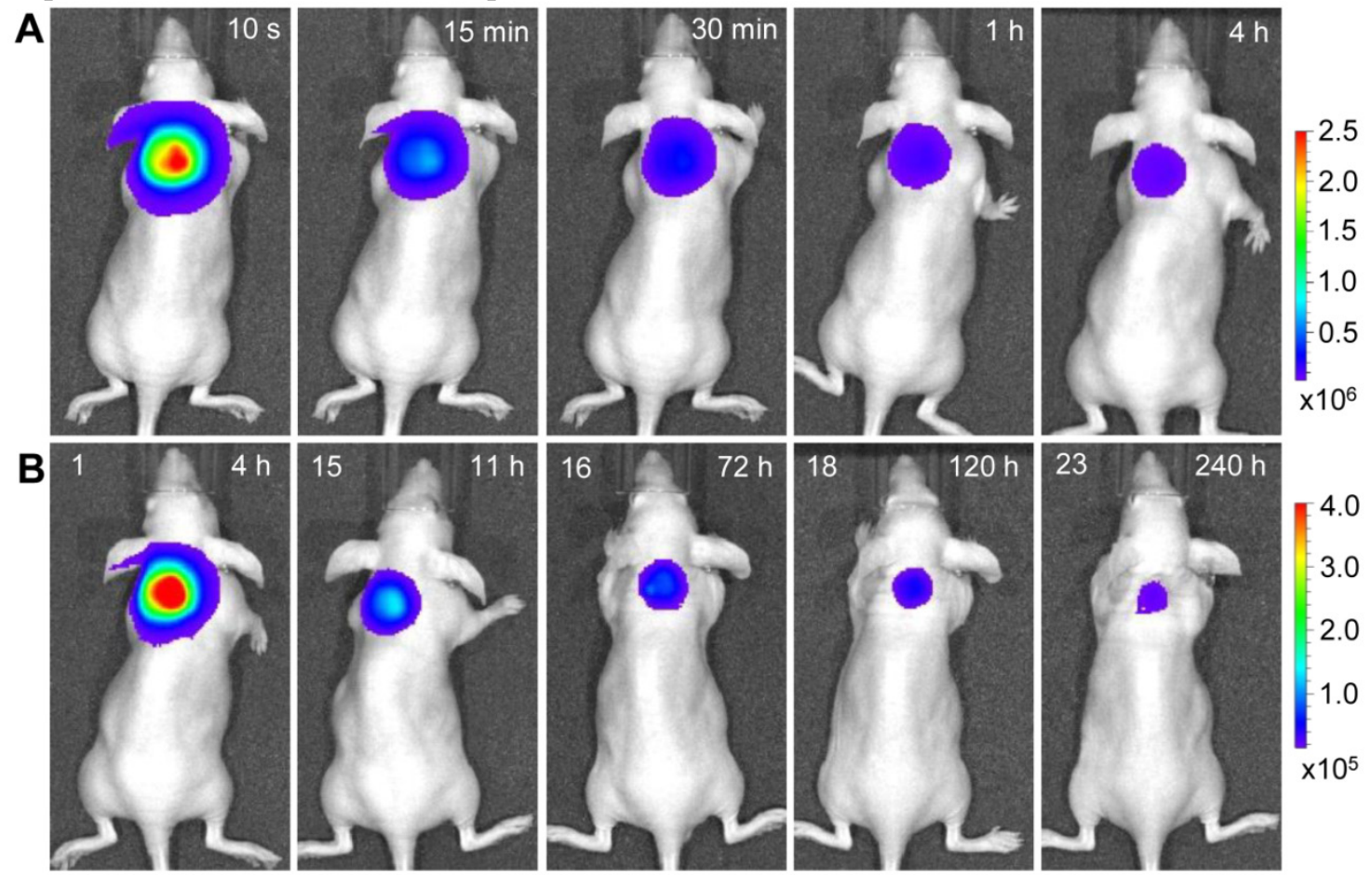

Figure 4. Acquisition of NIR persistent luminescence and NIR PSPL signals from PEI-LGO:Cr nanoparticles-labeled 4TI cells that were subcutaneously injected onto the back of a nude mouse. Before the injection, the labeled $4 \mathrm{TI}$ cells $\left(\sim 2.5 \times 10^{7}\right.$ cells; fixed) were irradiated by a $254 \mathrm{~nm}$ UV lamp for 15 min. (Row A) NIR persistent luminescence images taken within $4 \mathrm{~h}$ after the injection. The intensity of the NIR signals decreased gradually over the time. From $4 \mathrm{~h}$ to II h, the mouse was exposed every 30 min to a white LED flashlight (for I5 s) for a total of I5 times to elicit NIR PSPL signals. From $72 \mathrm{~h}$ to $240 \mathrm{~h}$, the mouse was irradiated daily by the LED flashlight (for $15 \mathrm{~s}$ ) for an additional 8 times. (Row B) Images of NIR PSPL taken immediately $(\sim 10 \mathrm{~s}$ delay) after I, 15, 16, 18, and 23 times of LED stimulation. The number at the upper left corner of each image in (Row B) indicates the stimulation times. The time at the upper right corner of each image indicates the time after the cell injection. All images were acquired on an IVIS imaging system in the bioluminescence mode with an exposure time of $2 \mathrm{~min}$.

Figure 5. Stimulation and acquisition of NIR PSPL signals from PEI-LGO:Cr nanoparticles inoculated into the brain of a nude mouse. The PEI-LGO:Cr nanoparticles $(\sim 25 \mu \mathrm{g})$ were irradiated by a $254 \mathrm{~nm}$ UV lamp for $15 \mathrm{~min}$ before the inoculation. At the $6 \mathrm{~h}(\mathrm{~A}), 72 \mathrm{~h} \mathrm{(B)}$, and $120 \mathrm{~h}$ (C) time points, the mouse head was illuminated by a white LED flashlight for $15 \mathrm{~s}$, and then imaged (I min delay) on an IVIS imaging system in the bioluminescence mode with an exposure time of $2 \mathrm{~min}$.

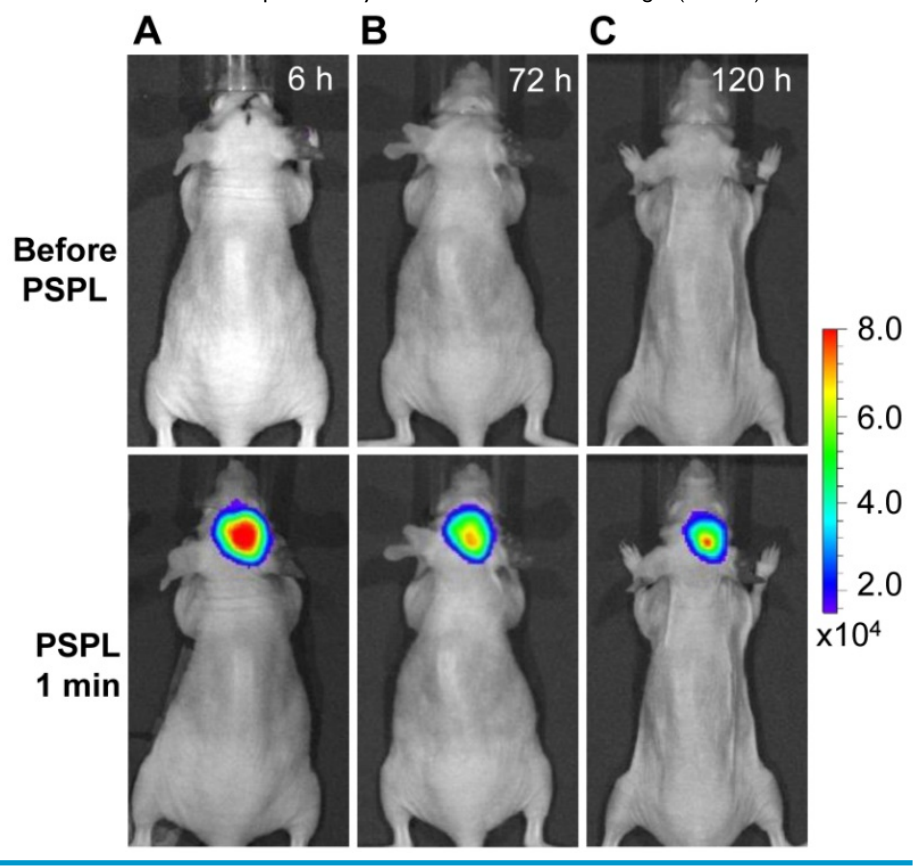


Cell tracking and active tumor targeting. The results in Figure 4 and Figure 5 clearly shows that a white LED flashlight can act as an external remote control to repeatedly recover the NIR signals from the UV pre-irradiated LGO:Cr nanoparticles in vivo. This capability is important for long-term cell tracking because tagged cells migrating to lesions need to be conveniently elicited to emit detectable luminescence signals. We performed a proof-of-concept cell tracking study by intravenously injecting PEI-LGO:Cr nanoparticles bearing RAW264.7 cells into 4T1 tumor models $(n=3)$, and monitoring the cell migration for 3 days on an IVIS imaging system in the bioluminescence mode, as shown in Figure 6A-C. Before the injection, UV pre-irradiated PEI-LGO:Cr nanoparticles were incubated with RAW264.7 cells in the dark for $48 \mathrm{~h}$. Since the particles had already experienced $48 \mathrm{~h}$ decay before the injection, the initial NIR persistent luminescence signal in vivo was too low to be detected by the IVIS system (top image in Figure 6A). However, intense NIR PSPL signals can be elicited by short-time (15 s) white LED stimulation. Imaging at 30 min (bottom image in Figure 6A) revealed NIR PSPL signals throughout the mouse body. At $72 \mathrm{~h}$ however, intense signals were found in the tumor area (bottom image in Figure 6C). Such a tropism is expected because macrophages are an important player in tumor proliferation and angiogenesis, and are heavily recruited to tumor sites [35], as was observed in previous reports by us [36] and others [37]. The cell tracking window of $72 \mathrm{~h}$ is about 1-2 orders of magnitude longer than that obtained in previous observations

\section{Cell Tracking}

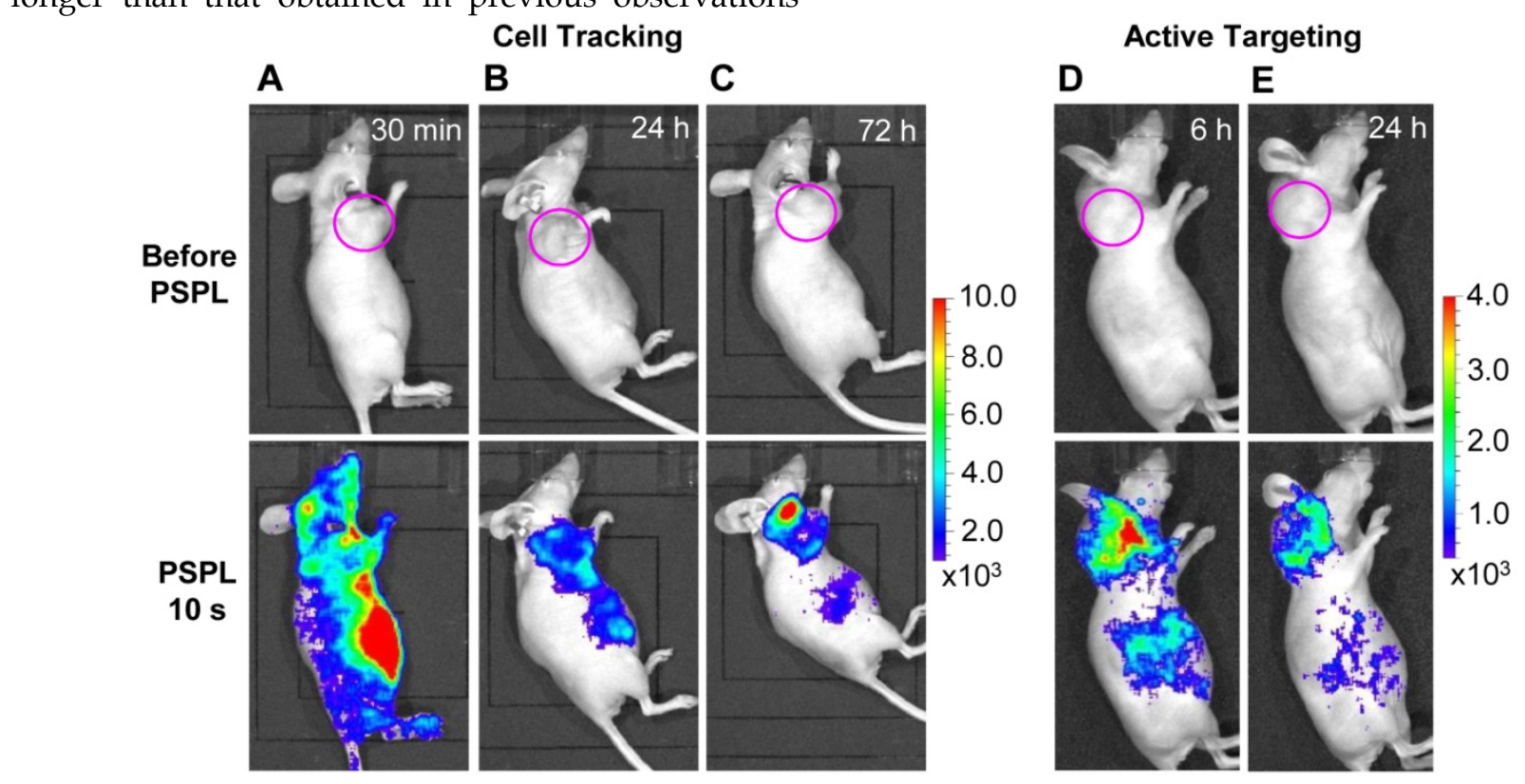

$(0.5-7.5 \mathrm{~h})[17,20]$. After the $72 \mathrm{~h}$ imaging, the animals were sacrificed. The tumors as well as major organs, including the liver, lung, spleen, kidney, heart and brain, were collected, UV-irradiated, and then subjected to ex vivo imaging study on an IVIS imaging system. Consistent with the in vivo results, the strongest NIR persistent luminescence signals were found in the tumors (Supplementary Material: Figure S11). The tumors were also fixed, sectioned, and examined on a LV200 bioluminescence microscope. The microscopy study showed inhomogeneous but strong persistent luminescence in the tumors (Supplementary Material: Figure S12).

Besides cell tracking, we also used LGO:Cr nanoparticles as tags of targeting molecules for cancer imaging. For this purpose, the LGO:Cr nanoparticles were first modified using polyvinylpyrrolidone (PVP) and human serum albumin (HSA), and then conjugated with $c($ RGDyK). $c($ RGDyK) is a small peptide with high affinity toward integrin $\alpha_{v} \beta_{3}$, a tumor biomarker overexpressed on tumor vasculature and tumor cells of various types $[38,39]$. Figures $6 \mathrm{D}$ and $6 \mathrm{E}$ show a tumor targeting study where c(RGDyK)-conjugated LGO:Cr nanoparticles were intravenously injected into 4T1 tumor bearing mice (n $=3$ ). Good accumulation of the nanoparticles in the tumors was observed (bottom images in Figures 6D and $6 \mathrm{E}$ ). The in vivo observation was consistent with the ex vivo imaging study, which showed strong NIR persistent luminescence signals in the tumors (Supplementary Material: Figure S13).

Figure 6. In vivo cell tracking and active tumor targeting with LGO:Cr nanoparticles. (A-C) Tracking the migration of PEI-LGO:Cr nanoparticles-labeled RAW264.7 cells in subcutaneous 4TI tumor model. The PEI-LGO:Cr nanoparticles were UV pre-irradiated by a $254 \mathrm{~nm}$ UV lamp for $15 \mathrm{~min}$, and then loaded into RAW264.7 cells for a $48 \mathrm{~h}$ incubation. The labeled RAW264.7 cells $\left(\sim 1.0 \times 10^{6}\right.$ cells) were intravenously injected. Whole body luminescence images were acquired before and after illuminating the mouse by a white LED flashlight (for I5 s) at (A) 30 min, (B) $24 \mathrm{~h}$, and (C) $72 \mathrm{~h}$ after the cell injection. (D,E) Active tumor targeting with c(RGDyK)-conjugated LGO:Cr nanoparticles in subcutaneous 4TI tumor model. The c(RGDyK)-LGO:Cr nanoparticles were irradiated by a $254 \mathrm{~nm}$ UV lamp for 15 min before the intravenous injection. Luminescence images were acquired before and after illuminating the mouse by a LED flashlight $($ for $15 \mathrm{~s}$ ) at (D) $6 \mathrm{~h}$ and $(\mathrm{E}) 24 \mathrm{~h}$ after the cell injection. The tumor sites are indicated by pink circles. All images were acquired on an IVIS imaging system in the bioluminescence mode with an exposure time of $3 \mathrm{~min}$. 


\section{Conclusions}

We have developed a novel type of optical nanoprobes, photostimulable LGO:Cr NIR persistent luminescent nanoparticles, which allows for ultrasensitive ( $\sim 5$ cells), deep-tissue, and long-term tracking (>3 days) of cells and molecule objects in vivo. This LGO:Cr nanoparticles-based imaging technique can be conveniently used on popular imaging systems such as the IVIS series to improve the quality and capacity of optical imaging, and holds the following promising prospects. Firstly, for cell tracking, LGO:Cr nanoparticles offer sensitivity and penetration depths that are comparable or superior to bioluminescence imaging, which is the most sensitive optical imaging method to date. Unlike bioluminescence-based cell tracking that requires tedious and sometimes challenging transfection to label cells as well as repeated injection of enzyme substrates, cell labeling with LGO:Cr nanoparticles demands no more than simple incubation. In addition, LGO:Cr nanoparticles-labeled cells can be examined histologically, an option that is not possible with bioluminescence-based cell tracking because luciferase proteins cannot survive the process of tissue fixation. Secondly, the LGO:Cr nanoparticles-based imaging allows one to "see" through a mouse body. This capacity is not possible with conventional fluorescence imaging, and the underlying impact of this advance can be tremendous. Thirdly, our results suggest great promise of LGO:Cr nanoparticles in cell assays such as flow cytometry and immunostaining. The advantages, aside from a clean background, also include the exceptional photo- and chemical-stability of LGO:Cr nanoparticles.

\section{Supplementary Material}

Figures S1-S13.

http://www.thno.org/v04p1112s1.pdf

\section{Abbreviations}

ESCs: murine embryonic stem cells; f-luc: firefly luciferase; HSA: human serum albumin; LED: light emitting diode; LGO:Cr: $\mathrm{LiGa}_{5} \mathrm{O}_{8}: \mathrm{Cr}^{3+}$; NIR: near-infrared; PBS: phosphate buffered saline; PEI: polyethylenimine; PSPL: photostimulated persistent luminescence; PVP: polyvinylpyrollidone; QDs: quantum dots; TEM: transmission electron microscope; UV: ultraviolet.

\section{Acknowledgments}

This work was supported by a NSF CAREER award (DMR-0955908, Z.W.P.), an NCI/NIH R00 grant (5R00CA153772, J.X.), the Intramural Research Program of NIBIB, NIH (X.C.), a NIH R01 grant (R01HL093339, L.C.W.), and a NIH P41 grant
(GM103390, L.C.W.). We thank Rick Tarleton for allowing us to use the IVIS Lumina II imaging system and Mary Ard for TEM imaging. We also thank Just Nanotech Co., Ltd. (Taiwan) for wet grinding the nanoparticles.

\section{Competing Interest}

The authors have declared that no competing interest exists.

\section{References}

1. Massoud TF, Gambhir SS. Molecular imaging in living subjects: seeing fundamental biological processes in a new light. Genes Dev. 2003; 17: 545-80.

2. Willmann JK, van Bruggen N, Dinkelborg LM, Gambhir SS. Molecular imaging in drug development. Nat Rev Drug Discov. 2008; 7: 591-607.

3. So MK, Xu C, Loening AM, Gambhir SS, Rao J. Self-illuminating quantum dot conjugates for in vivo imaging. Nat Biotechnol. 2006; 24: 339-43.

4. Choy G, O'Connor S, Diehn FE, Costouros N, Alexander HR, Choyke $\mathrm{P}$, Libutti SK. Comparison of noninvasive fluorescent and bioluminescent small animal optical imaging. Biotechniques. 2003; 35: 1022-30.

5. Luo SL, Zhang EL, Su YP, Cheng TM, Shi CM. A review of NIR dyes in cancer targeting and imaging. Biomaterials. 2011;32: 7127-38.

6. Panchuk-Voloshina N, Haugland RP, Bishop-Stewart J, Bhalgat MK, Millard PJ, Mao F, Leung WY, Haugland RP. Alexa dyes, a series of new fluorescent dyes that yield exceptionally bright, photostable conjugates. J Histochem Cytochem. 1999; 47: 1179-88.

7. Chalfie M, Tu Y, Euskirchen G, Ward WW, Prasher DC. Green fluorescent protein as a marker for gene expression. Science. 1994; 263: 802-5.

8. Shu X, Royant A, Lin MZ, Aguilera TA, Lev-Ram V, Steinbach PA, Tsien RY. Mammalian expression of infrared fluorescent proteins engineered from a bacterial phytochrome. Science. 2009; 324: 804-7.

9. Filonov GS, Piatkevich KD, Ting LM, Zhang J, Kim K, Verkhusha VV. Bright and stable near-infrared fluorescent protein for in vivo imaging. Nat Biotechnol. 2011; 29: 759-63.

10. Resch-Genger $U$, Grabolle $M$, Cavaliere-Jaricot $S$, Nitschke $R$, Nann T. Quantum dots versus organic dyes as fluorescent labels. Nat Methods. 2008; 5 : 763-75.

11. Michalet X, Pinaud FF, Bentolila LA, Tsay JM, Doose S, Li JJ, Sundaresan G, Wu AM, Gambhir SS, Weiss S. Quantum dots for live cells, in vivo imaging, and diagnostics. Science. 2005; 307: 538-44.

12. Gao JH, Chen XY, Cheng Z. Near-infrared quantum dots as optical probes for tumor imaging. Curr Top Med Chem. 2010; 10: 1147-57.

13. Aswathy RG, Yoshida Y, Maekawa T, Kumar DS. Near-infrared quantum dots for deep tissue imaging. Anal Bioanal Chem. 2010; 397: 1417-35.

14. Wang F, Banerjee D, Liu YS, Chen XY, Liu XG. Upconversion nanoparticles in biological labeling, imaging, and therapy. Analyst. 2010; 135: 1839-54.

15. Zagorovsky K, Chan WCW. Illuminating the deep. Nat Mater. 2013; 12: 285-7.

16. Frangioni JV. Self-illuminating quantum dots light the way. Nat Biotechnol. 2006; $24: 326-8$.

17. de Chermont QLM, Chanéac C, Seguin J, Pellé F, Maîtrejean S, Jolivet JP, Gourier D, Bessodes M, Scherman D. Nanoprobes with near-infrared persistent luminescence for in vivo imaging. Proc Natl Acad Sci USA. 2007; 104: 9266-71

18. So MK, Loening AM, Gambhir SS, Rao JH. Creating self-illuminating quatum dot conjugates. Nat Protoc. 2006; 1: 1160-4.

19. Xiong LQ, Shuhendler A, Rao JH. Self-luminescing BRET-FRET near-infrared dots for in vivo lymph-node mapping and tumor imaging. Nat Commun. 2012; 3: 1193.

20. Abdukayum A, Chen JT, Zhao Q, Yan XP. Functional near infrared-emitting $\mathrm{Cr}^{3+} / \mathrm{Pr}^{3+}$ co-doped zinc gallogermanate persistent luminescent nanoparticles with super-long afterglow for in vivo targeted bioimaging. J Am Chem Soc. 2013; 135: 14125-33.

21. Yen WM, Shionoya S, Yamamoto H. Phosphor handbook, 2nd ed. Boca Raton, FL: CRC Press; 2007.

22. Pan $Z W, L u$ YY, Liu F. Sunlight-activated long-persistent luminescence in the near-infrared from $\mathrm{Cr}^{3+}$-doped zinc gallogermanates. Nat Mater. 2012; 11: 58-63.

23. Rogers WJ, Meyer $\mathrm{CH}$, Kramer CM. Technology insight: in vivo cell tracking by use of MRI. Nat Clin Pract Cardiovasc Med. 2006; 3: 554-62.

24. Liu F, Yan WZ, Chuang YJ, Zhen ZP, Xie J, Pan ZW. Photostimulated near-infrared persistent luminescence as a new optical read-out from $\mathrm{Cr}^{3+}$-doped $\mathrm{LiGa}_{5} \mathrm{O}_{8}$. Sci Rep. 2013; 3: 1554

25. Szymczak $\mathrm{H}$, Wardzynska M, Mylnikova IE. Optical spectrum of $\mathrm{Cr}^{3+}$ in the spinel LiGa5O $\mathrm{O}_{8}$ J Phys C: Solid State Phys. 1975; 8: 3937-43.

26. Canton I, Battaglia G. Endocytosis at the nanoscale. Chem Soc Rev. 2012; 41: 2718-39.

27. Cheng ZL, Al Zaki A, Hui JZ, Muzykantov VR, Tsourkas A. Multifunctional nanoparticles: cost versus benefit of adding targeting and imaging capabilities. Science. 2012; 338: 903-10. 
28. O'Dell, BL, Sunde RA. Handbook of nutritionally essential mineral elements. New York: CRC Press; 1997.

29. Di Bona KR, Love S, Rhodes NR, McAdory D, Sinha SH, Kern N, Kent J, Strickland J, Wilson A, Beaird J, et al. Chromium is not an essential trace element for mammals: effects of a "low-chromium" diet. J Biol Inorg Chem. 2011; 16: 381-90.

30. Hoffer P. Status of gallium-67 in tumor detection. J Nucl Med. 1980; $21: 394-8$.

31. Contag $\mathrm{CH}$, Bachmann $\mathrm{MH}$. Advances in in vivo bioluminescence imaging of gene expression. Annu Rev Biomed Eng. 2002; 4: 235-60.

32. Rabinovich BA, Ye Y, Etto T, Chen JQ, Levitsky HI, Overwijk WW, Cooper LJ, Gelovani J, Hwu P. Visualizing fewer than 10 mouse T cells with an enhanced firefly luciferase in immunocompetent mouse models of cancer. Proc Natl Acad Sci USA. 2008; 105: 14342-6.

33. Walling MA, Novak JA, Shepard JR. Quantum dots for live cell and in vivo imaging. Int J Mol Sci. 2009; 10: 441-91.

34. Sutton EJ, Henning TD, Pichler BJ, Bremer C, Daldrup-Link HE. Cell tracking with optical imaging. Eur Radiol. 2008; 18: 2021-32.

35. Condeelis J, Pollard JW. Macrophages: obligate partners for tumor cell migration, invasion, and metastasis. Cell. 2006; 124: 263-6.

36. Xie J, Wang J, Niu G, Huang J, Chen K, Li X, Chen X. Human serum albumin coated iron oxide nanoparticles for efficient cell labeling. Chem Commun. 2010; 46: 433-5.

37. Green CE, Liu T, Montel V, Hsiao G, Lester RD, Subramaniam S, Gonias SL, Klemke RL. Chemoattractant signaling between tumor cells and macrophages regulates cancer cell migration, metastasis and neovascularization. PLoS One. 2009; 4: e6713

38. Cai W, Chen X. Multimodality molecular imaging of tumor angiogenesis. J Nucl Med. 2008; 49: S113-28.

39. Hood JD, Cheresh DA. Role of integrins in cell invasion and migration. Nat Rev Cancer. 2002; 2: 91-100. 